[0212-7199(2003) 20: 1 ; pp 28-30]
ANALES DE MEDICINA INTERNA Copyright $\odot 2003$ ARAN EDICIONES, S.L.

AN. MED. INTERNA (Madrid) AN. MED. INTERNA (Madrid)
Vol. 20, N. ${ }^{\circ}$ 1, pp. $28-30,2003$

\section{Enfermedad de Fabry asociada a artritis reumatoide. Una encrucijada multisistémica}

\author{
N. ARIAS MARTÍNEZ, F. J. BARBADO HERNÁNDEZ, G. PÉREZ MARTÍN, \\ C. PÉREZ DE AYALA ${ }^{1}$, V. CASAL ESTEBAN, J. J. VÁZQUEZ RODRÍGUEZ \\ Servicios de Medicina Interna y 'Reumatología. Hospital Universitario La Paz. \\ Universidad Autónoma. Madrid
}

\author{
FABRY'S DISEASE ASSOCIATED WITH RHEUMATOID ARTHRITIS \\ A MULTISYSTEM CROSSROAD
}

\section{RESUMEN}

La enfermedad de Fabry es una alteración congénita poco frecuente, del metabolismo de los glucoesfingolípidos, en la cual existe un déficit de la enzima alfa galactosidasa A. Se trasmite de modo recesivo ligada al $\mathrm{X}$ por lo que las manifestaciones clínicas son más severas en varones hemicigóticos. El diagnóstico se lleva a cabo mediante la determinación de los niveles del enzima alfa-galactosidasa.

En la literatura se han descrito varios casos de enfermedad de Fabry asociada a enfermedades del tejido conectivo, pero no se ha encontrado ningún caso coexistiendo con artritis reumatoide.

Presentamos un caso de una paciente con enfermedad de Fabry que, posteriormente se diagnosticó de artritis reumatoide. El diagnóstico de sospecha fue fundamental ya que el carácter multisistémico de las manifestaciones clínicas de ambas enfermedades hace que sea difícil no atribuir nuevos signos a la enfermedad de Fabry.

El acúmulo de lípidos provoca un estímulo antigénico prolongado que es capaz de formar inmunocomplejos. Esta es la hipótesis patogénica por la que creemos que la enfermedad de Fabry se asocia a enfermedades autoinmunes.

PALABRAS CLAVE: Enfermedad de Fabry. Artritis reumatoide. Enfermedades multisistémicas.

\begin{abstract}
Fabry's disease is a rare congenic disorder of glycosphingolipid catabolism resulting from deficient activity of the alpha galactosidasa. Is an X-linked disorder and in hemizygous males the activity of this enzyme is very low, resulting in severe manifestations. Fabry disease is confir med by the lack alfa-galactosidase in serum.

In the literature have been reported a few cases of coexistant Fabry's disease and conective disorders, but there is not cases of rheu matoid arthritis coexistant.

This report describes a case of a female with Fabry's disease who vas subsequently diagnosed with reumatoid arthritis. The suspect diag nosis was very important because the two disorders are multisystem and new simptoms culd be attributed to Fabry's disease.

The accumulation of lipids may results in mumerous pathogenic autoantibodies, which could make inmunocomplex. This is the potential pathogenic mechanisms explaining the association between Fabry's disease and autoinmune diseases.
\end{abstract}

KEY WORDS: Fabry's disease. Rheumatoid artritis. Multisistemic disorders.

Arias N, Barbado FJ, Pérez Martín G, Pérez de Ayala C, Casal V, Vázquez JJ. Enfermedad de Fabry asociada a artritis reumatoide. Una encrucijada multisistémica. An Med Interna (Madrid) 2003; 20: 28-30.

\section{INTRODUCCIÓN}

La enfermedad de Fabry es una entidad clínica infrecuente, que se produce por el acúmulo de trihexosidogalactosilglucosil ceramido debido a un déficit congénito de alfa galactosidasa. La herencia se trasmite de modo recesivo ligada al cromosoma $\mathrm{X}$, siendo las manifestaciones clínicas más severas en varones hemicigóticos. Se caracteriza por angioqueratomas, hipohidrosis, opacificación corneal, alteraciones del cristalino, acroparestesias y lesiones vasculares que pueden afectar a distintos órganos: riñón, corazón y cerebro (1-3).

Se han descrito en la literatura varios casos de enfermedad de Fabry coexistiendo con enfermedades del tejido conectivo, en concreto con lupus eritematoso sistémico (4-7) y con un síndrome de Raynaud, eritermalgia y acrodinia (8).

Presentamos una asociación no descrita previamente en la que se diagnosticó una artritis reumatoide en una paciente con enfermedad de Fabry.

\section{CASO APORTADO}

Mujer de 45 años con antecedentes familiares de padre muerto a los 49 años por insuficiencia renal crónica y con episodios de dolor

Trabajo aceptado: 6 de noviembre de 2001

Correspondencia: Natividad Arias Martínez. Avenida del Ejército, 11-D; 1-B. 19004 Guadalajara 
en región distal de las manos, dos hijos varones diagnosticados de enfermedad de Fabry y una hija sana.

Desde los 8 años tiene episodios de dolor intenso en las falanges de los dedos de las extremidades inferiores y desde los 18 años, de forma recurrente, dolor intenso y lancinante en los dedos de las manos que, en algunas ocasiones, se ha acompañado de febrícula. En la exploración física se observó amiotrofia y coloración cutánea parduzca en región tenar e hipotenar.

Entre los datos analíticos se observó $\mathrm{Hb} 10,9 \mathrm{~g} / \mathrm{dl}$, Htc $36 \%$, VCM 78,CHCM 23, leucocitos 6760 con fórmula normal, plaquetas 257000, VSG 8, haptoglobina 247, Fe sérico $26 \mathrm{mcg} / \mathrm{dl}$, transferrina $324 \mathrm{mg} / \mathrm{dl}$ y ferritina $3 \mathrm{ng} / \mathrm{dl}$. Las determinaciones de bioquímica sérica, coagulación, proteinograma y orina elemental que fueron normales. Se determinó la alfa galactosidasa $0,33 \mathrm{mU} / \mathrm{ml}$ y beta galactosidasa $1,19 \mathrm{mU} / \mathrm{ml}$ en suero y en leucocitos la alfa galactosidasa con un resultado de 256 nmoles por min. Por g. prot. (controles de 392, 675 y 729 respectivamente) y el cociente alfa galactosidasa / beta hexosaminidasa con un resultado de 16,6 nmoles por min. por $\mathrm{g}$. prot. (controles de 23,8, 22,5, 34,8 respectivamente).

El estudio radiológico de tórax y abdomen y la ecografía abdominal no pusieron de manifiesto ninguna alteración. El EMG demostró signos muy leves de neuropatía periférica sensitiva de predominio desmielinizante.

Fue diagnosticada de enfermedad de Fabry y se trató de modo sintomático con carbamacepina.

Realizó revisiones periódicas en la consulta y seis años más tarde acude por episodios de dolor, hinchazón e impotencia funcional en articulaciones simétricas (rodillas, tobillos, carpos y $\mathrm{MCF}$ ), con rigidez matutina y febrícula. En la exploración destacó la presencia de signos inflamatorios en codo izquierdo, ambos carpos y rodillas, con livedo reticularis en miembros inferiores.

Entre los datos analíticos se observó anemia microcítica mormocrómica, con aumento de la fosfatasa alcalina y GGT. Asimismo, tenía elevados reactantes de fase aguda (VSG y fibrinógeno). Hierro sérico $42 \mathrm{mcg} / \mathrm{dl}$, transferrina $192 \mathrm{mg} / \mathrm{dl}$, saturación de transferrina $77 \%$ y ferritina $73 \mathrm{ng} / \mathrm{dl}$. Estudio inmunológico: factor reumatoide positivo, ANA negativo, inmunoglobulinas y complemento normales, PCR de 12 mg/dl y ASLO <200. Los estudios microbiológicos fueron negativos. El estudio radiológico reveló erosiones en articulaciones metacarpofalángicas proximales, con pinzamiento de la linea articular en el carpo izquierdo (Fig. 1). Mediante artrocentesis de las articulaciones de ambas rodillas, se obtuvo un líquido de características inflamatorias (3500 leucos $/ \mathrm{mm}^{3}, 25 \% \mathrm{PMN}$ ), cuyo cultivo fue estéril. Se diagnosticó de artritis reumatoide al cumplir 4 de 7 criterios del ARA (artritis de más de 3 áreas articulares, simétrica, erosiva, afectando a las articulaciones de la mano y con factor reumatoide

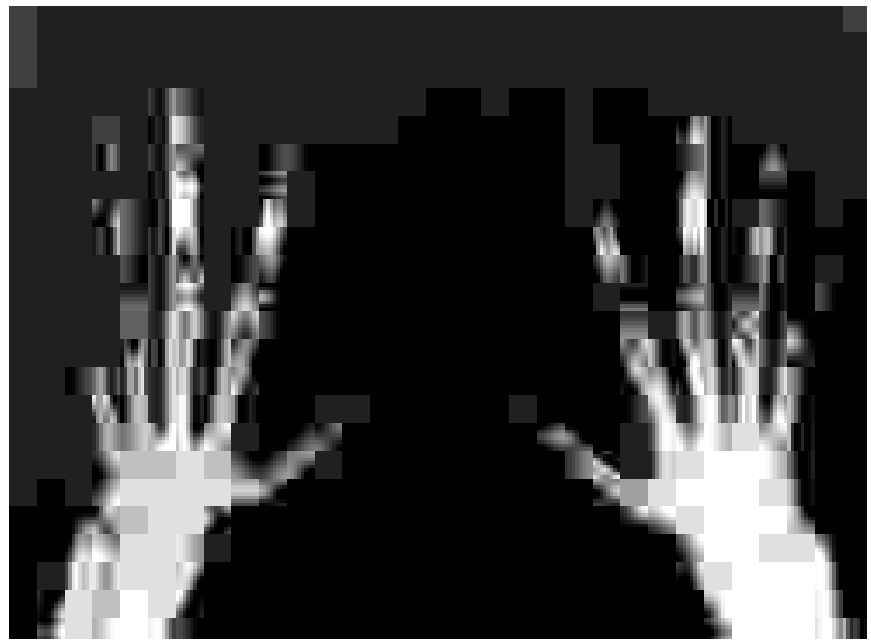

Fig. 1. Erosiones en articulaciones metacarpofalángicas proximales, con pinzamiento de la línea articular en el carpo izquierdo. positivo). Fue tratada con esteroides y metotrexate, con moderada mejoría del cuadro articular. Ha realizado revisiones periódicas durante estos últimos dos años, durante los cuales ha precisado mantener el tratamiento inmunosupresor.

\section{DISCUSIÓN}

La enfermedad de Fabry es una alteración congénita del metabolismo de los glucoesfingolípidos, con una prevalencia de 1/40.000. Existe un déficit de la enzima alfa galactosidasa A que se codifica en el brazo largo del cromosoma $\mathrm{X}$, lo que condiciona el acúmulo de trihexosidogalactosilglucosil ceramido (1-3).

Se trata de una enfermedad multisistémica, cuyas manifestaciones clínicas son más severas en varones hemicigóticos. Aparecen angioqueratomas, disminuye o desaparece la sudoración, hay opacificación corneal y lesiones en el cristalino con tortuosidad de los vasos conjuntivales y retinianos, dolor en manos, pies y zona proximal de las extremidades, dolor abdominal, bronquitis crónica, linfedema sin hipoproteinemia, diarrea, osteoporosis, retraso del crecimiento y de la pubertad y afectación vascular renal, cardiaca y cerebral, que se manifiesta como insuficiencia renal, proteinuria, hipertensión arterial, insuficiencia cardiaca e isquemia (10). Asimismo aparece poliartritis con multiples osificaciones de las entesis y algunas erosiones intra y extraarticulares (10) y existen microcristales en el citoplasma y las mitocondrias de las células de la sinovial (11).

El diagnóstico de sospecha es clínico y se confirma demostrando la disminución de la actividad de la alfa galactosidasa en plasma, leucocitos o linfoblastos-fibroblastos en cultivo (1-3). Una vez confirmada la enfermedad de Fabry el diagnóstico de alguna otra enfermedad multisistémica es dificil, ya que los síntomas se pueden solapar y confundir, atribuyéndose a la primera (4).

Se ha revisado la literatura (Medline entre los años 1978 y 1999), y se han encontrado descritos cinco casos de enfermedad de Fabry asociada a enfermedades del tejido conectivo. En cuatro casos se trataba de lupus eritematoso sistémico (47) y en el quinto de un fenómeno de Raynaud y acrodinia (8). En tres de ellos, es de destacar que el que se tratase de pacientes con enfermedad de Fabry ya conocida, dificultó el diagnóstico de la enfermedad del tejido conectivo concomitante $(5,6,8)$. Esto fue debido a que se pensó que las manifestaciones clínicas de estas enfermedades fueran causadas por la enfermedad de Fabry. Sin embargo, en los dos casos restantes, ante la sospecha de la existencia de un lupus eritematoso sistémico, se llevó a cabo una biopsia renal. En dichas biopsias, además de las lesiones propias de la nefropatía lúpica, se puso de manifiesto alteraciones compatibles con enfermedad de Fabry, que se confirmó con determinaciones bioquímicas $(4,7)$.

En el caso que presentamos el diagnóstico se sospechó por la clinica de dolor en las extremidades, así como por los antecedentes de enfermedad de Fabry en sus hijos, en uno de los cuales la forma de presentación inicial fue un episodio de priapismo (12), y se confirmó mediante métodos bioquímicos.

En nuestro caso, inicialmente, la paciente presentaba dolores recurrentes en extremidades debidos a la enfermedad de Fabry, sin embargo no se objetivaron signos inflamatorios ni erosiones en las radiografías, por lo que podemos suponer que 
no se trató de un brote de artritis reumatoide. Sin embargo, en la evolución posterior se puso de manifiestó un cambio en la sintomatología, apareciendo rigidez y signos inflamatorios en la exploración física de las articulaciones, por lo que realizó un estudio inmunológico y radiológico y se diagnosticó de artritis reumatoide.

En la casuística revisada (Medline entre los años 1978 y 1999) no hemos encontrado descrita la asociación de enfermedad de Fabry y de artritis reumatoide, aunque sí existe afectación articular en la enfermedad de Fabry por depósito de lípidos en la piel y en los compartimentos sinoviales. Se produce así un alargamiento de la cápsula articular y los tendones, con osificación de las entesis en la inserción de las estructuras fibrosas y algunas erosiones intra y extraarticulares (9). Estas erosiones son infrecuentes y se diferencian de las que aparecen en la artritis reumatoide, en la que son yuxtaarticulares.

El diagnóstico es similar a otros casos de artritis reumatoide, si bien es necesario un alto índice de sospecha debido a que las manifestaciones clínicas de ambas entidades pueden solaparse.

El tratamiento aplicado no fue diferente al de otros casos de artritis reumatoide, basándose en esteroides y metotrexate con lo que se consiguió la mejoría parcial del cuadro inflamatorio articular.

La hipótesis patogénica por la cual la enfermedad de Fabry se asocia a enfermedades autoinmunes tales como la artritis reumatoide, o el lupus (4), podría ser el acúmulo de lípidos que provoca un estímulo antigénico prolongado, de manera que aparecen numerosos anticuerpos con capacidad patogénica, y que son capaces de formar inmunocomplejos, como por ejemplo los existentes en el lupus eritematoso sistémico.

Así pues, podemos concluir que la presencia de una enfermedad de Fabry puede tener influencia sobre el posterior desarrollo de una enfermedad sistémica autoinmune del tejido conectivo.

\section{Bibliografía}

1. McGovern MM. Enfermedades por depósito lisosómico, en Principios de Medicina Interna de Harrison. 14 Ed. Mc.Graw-Hill. Interamericana Ed. 1998. Madrid. 2467-75.

2. Brady RO, Gal AE, Bradley RM, Martensson E, Warshaw AL, Laster I: Enzymatic defect in Fabry's disease. Cereidetrihexosidase deficiency. N Engl J Med 1967: 276: 1163-7.

3. Burda CD, Winder PR: Angiokeratoma corporis diffusum universale (Fabry's disease) in female subjects. Am J Med 1967; 42: 293-301.

4. Rahman P, Gladman DD, Wither J and Silver MD. Coexistence of Fabry's disease and systemic lupus erythematosus. Clin Exp Rheumatol 1998; 16:475-478.

5. Loeb H, Jonniaux G, Tondeur M: Etude clinique, biochimique et untrastructurelle de la maladie de Fabry chez l'enfant. Helv Paediat Acta 1968; 3: 269-86.

6. Rosenmann E, Kobrin I, Cohen T: Kidney involvement en SLE and Fabry's disease. Nephron 1983; 34: 180-4.
7. Majima K, Ishizaki T, Inoue T: A case of Fabry's disease associated with lupus nephritis. Nippon Jinzo Gakkai Shi 1992; 34:1189-94.

8. Cailleux N, Levesque H, Joly P, Thomine E, Courtois H. Acromélalgies de l'enfant a propos d'une observation révélatrice de maladie de Fabry. J Mal Vasc 1995; 20; 142-145.

9. Fischer E. Fabry disease, a disease with rheumatic aspects: radiology of soft tissue and bone changes in the hand. Z Rheumatol 1986; 45: 36-41.

10. Mateos Sánchez A, Sanz Ortega F, Becares Lozano M, Ruiz Beltrán R, Querol Prieto R, Hernández Hernández A, De Portugal Álvarez J. Enfermedad de Fabry: un estudio familiar. An Med Interna (Madrid) 1985; 2 : 281-288.

11. Delbarre F, Laoussadi S, Kahan A, Aubouy G. Rheumatism caused by lipid thesaurismosis. C R Seances Acad Sci D 1979; 288: 181-4.

12. García-Consuegra J, Padrón M, Jaureguizar E: Priapism and Fabry disease: a case report. Eur J Pediatr 1990; 149: 500-501. 